\title{
Die Interstate Commerce Commission
}

ENTSTEHUNG, ENTWiCKLUNG UND GegenWARTSSTAND DES REgULIERENDEN STAATSEINGRIFFS IN DEN USA AM BEISPIEL EINER REgULIERUNGSKOMMISSION

\author{
INAUGURAL-DISSERTATION \\ ZUR ERLANGUNG DES GRADES EINES \\ DOKTORS DER WIRTSCHAFTS- UNd SOZIALWISSENSCHAFTEN \\ am Fachbereich Politische Wissenschaft \\ Der Freien Universität BerLiN
}

VORGELEGT

VON

DiPl. Pol. HaNs Jürgen Kleinsteuber

Aus Lemgo

BERLIN 1975 
ISBN 978-3-476-99572-8

ISBN 978-3-476-99571-1 (eBook)

DOI 10.1007/978-3-476-99571-1

1. Gutachter: Prof. Dr. Gert von Eynern

2. Gutachter: Prof. Dr. Abraham Ashkenasi

Tag der mündlichen Prüfung: 4. Juli 1975 


\section{Inhalt}

1. Einleitung . . . . . . . . . . . . . 1

2. Okonomische und politische Faktoren bei der Entstehung der Interstate Commerce Commission .......... 5

2.1 Die Bedeutung der Eisenbahnen in der Geschichte der USA . . . 5

2.2 Die Entstehung des 'Eisenbahnproblems' . . . . . . . . 7

2.2.1 Duplizierungen im Eisenbahnstreckennetz . . . . . . . . 9

2.2.2 Der ruinöse Wettbewerb . . . . . . . . . . . . . . . 12

2.2.3 Der Verfall der Eisenbahntarife . . . . . . . . . . 13

2.3 Die Folgen des 'Eisenbahnproblems' . . . . . . . . . . 17

2.3.1 Lokale und ökonomische Diskriminierung . . . . . . . . . . 17

2.3.2 Versuchte Kartellabsprachen: Die Pools . . . . . . . . . . 20

2.4 Politische Positionen zur bundesstaatlichen Regulierung der Eisenbahnen . . . . . . . . . . . . . . 23

2.4.1 Die Granger-Bewegung und die ersten 'harten' Eisenbahnkommissionen . . . . . . . . . . . . . . . . 24

2.4 .2 Die Merchants . . . . . . . . . . . . . . . . . . . . 30

2.4.3 Die Railroad-Leaders . . . . . . . . . . . . . . . 33

2.5 Die parlamentarische Gründungsgeschichte der ICC . . . . 38

3. Zur Legislativgeschichte der ICC . . . . . . . . . . . . 44

3.1 Das Element der Kontinuität in der Geschichte der ICC . . . . . 44

3.2 Die Konsolidierung der ICC 1887-1917 . . . . . . . . . . 45

3.3 Die Kriegsverstaatlichungen der Eisenbahnen 1917-1920 . . . 49

3.4 Reprivatisierung, der Transportation Act und die ICC 1920-1929 51

3.5 Die Auswirkungen der Weltwirtschaftskrise und der Motor Carrier Act 1929-1940 . . . . . . . . . . . . . . . 54

3.6 Die ICC seit $1940 \ldots \ldots \ldots \ldots \ldots$

4. Die ICC: Institution und Verfahren . . . . . . . . . . 61

4.1 Die Institution . . . . . . . . . . . . . . . 61

4.1.1 Die wesentlichen Aufgaben der ICC . . . . . . . . . . 61

4.1 .2 Die ICC-Kommission . . . . . . . . . . . . 63 
4.1.3 Die Struktur der ICC . . . . . . . . . . . . . 65

4.1.4 Die Finanzausstattung der ICC . . . . . . . . . 68

4.1.5 Das Personal der ICC . . . . . . . . . . . . . . 70

4.2 Das Verfahren ............... . . 76

4.2.1 Die quasi-Funktionen der ICC . . . . . . . . . . . . 76

4.2.2 Allgemeine Prinzipien bei ICC-Verfahren . . . . . . . . 79

4.2.3 Die Rechtsgrundlage von ICC-Verfahren . . . . . . . . . . 83

4.2.4 Die Rolle der Examiners im ICC-Verfahren . . . . . . . . . 83

4.2.5 Die Bedeutung der Practitioners . . . . . . . . . . . 85

4.2.6 Eine Statistik der vor der ICC streitenden Parteien . . . . . . . 88

4.3 Die Vertretung des Eigeninteresses durch die ICC . . . . . . . 92

5. Die Stellung der ICC im politischen System der USA . . . . . . 97

5.1 Die ICC im Spannungsfeld von Kongreß und Präsident . . . . . 98

5.2 Die ICC und der Kongreß . . . . . . . . . . . . . . . 103

5.2.1 Kongreßausschüsse und die ICC . . . . . . . . . . . . . 104

5.2.2 Der Kongreß als Austragungsort von Regulierungsstreitigkeiten, . 106

5.2.3 Individuelle Interventionen von Kongreßabgeordneten . . . . . 107

5.3 Die ICC und der Präsident als Leiter der Exekutive . . . . . . . 109

5.3.1 Die politische Bedeutung von Einwirkungsmöglichkeiten des Präsidenten gegenüber der ICC . . . . . . . . . . . . 110

5.3.2 Die Interessen des Präsidenten gegenüber der ICC . . . . . . . . 113

5.3.3 Die Rolle des Department of Justice . . . . . . . . . . . 115

5.3.4 Das Department of Transportation als Gegengewicht zur ICC . . 117

5.3.5 Der Council of Economic Advisers . . . . . . . . . . 118

5.4 Die ICC und die rechtsprechende Gewalt . . . . . . . . 120

5.4.1 Das richterliche Prüfungsrecht in der Entwicklung der ICC . . . 120

5.4.2 Der Supreme Court als letztinstanzlicher Regulierer . . . . . . 123

6. Die ICC im Umfeld von Transportinteressen . . . . . . . . . 130

6.1 Die Unterstützung durch die Regulierten . . . . . . . . . . . 130

6.2 Die Kritik der Leistungsabnehmer . . . . . . . . . . . . 135

6.3 Technische und nicht-technische Kooperation mit der ICC . . 137

6.4 Die Bedeutung informeller Verfahrensweisen. . . . . . . . 140

6.5 Die ICC und der Konsument . . . . . . . . . . . . . . . 142

6.5.1 Die selbsterklärte Nichtzuständigkeit . . . . . . . . . . . . 142

6.5.2 Die Sonderstellung des Small Business . . . . . . . . . . 144

7. Die Regulierungstätigkeit der ICC und die wirtschaftlichen Folgen 146

7.1 Die Tarifregulierung durch die ICC . . . . . . . . . . . 147

7.1.1 Die Tarifpolitik der ICC . . . . . . . . . . . . . . 147

7.1.2 Zum Vergleich regulierter und nicht-regulierter Tarife . . . . 153

7.1.3 Die Bedeutung der Selbstregulierung durch die Rate Bureaus . . 154

7.2 Regulierung durch Kontrolle des Marktzugangs . . . . . . 158 
7.2.1 Die ökonomische Bedeutung des Lizenzzwangs . . . . . . . 158

7.2.2 Lizenzzwang und Bürokratisierung . . . . . . . . . . . . . 162

7.3 Die Vermachtung der Märkte als Folge der Regulierung . . . . . 164

7.4 Die ICC und die Verpflichtung auf eine nationale 'Transportpolitik 166

7.5 Das ungelöste 'nationale Transportproblem' und das Versagen der ICC . . . . . . . . . . . . . . . . . 171

8. Politisch-ökonomische Aspekte der Regulierung in den USA am Beispiel der ICC und weiterer Kommissionen . . . . . . . . 181

8.1 Historische Wurzeln der Regulierung . . . . . . . . . . . . . 181

8.2 Der Begriff der Regulierung . . . . . . . . . . . . . . 183

8.3 Die expansive Tendenz der Regulierung in den USA . . . . . . 190

8.4 Die unabhängige Kommission als regulierende Behörde . . . . . 193

8.5 Die Gewaltenhäufung in den unabhängigen Regulierungskommissionen . . . . . . . . . . . . . . . . . . 199

8.6 Die sozialen Kosten der Regulierung . . . . . . . . . . 202

Anmerkungen . . . . . . . . . . . . . . . 212

Literaturverzeichnis . . . . . . . . . . . . . . 243

Personenregister .................. . 254 


\section{Abkürzungen}

$\begin{array}{ll}\text { AAR } & \text { Association of American Railroads } \\ \text { AEC } & \text { Atomic Energy Commission } \\ \text { APA } & \text { Administrative Procedure Act } \\ \text { ATA } & \text { American Trucking Associations } \\ \text { AT \& T } & \text { American Telephone \& Telegraph } \\ \text { CAB } & \text { Civil Aeronautics Board } \\ \text { CEA } & \text { Council of Economic Advisers } \\ \text { COMET } & \text { Committee on Modern Efficient Transportation } \\ \text { DOJ } & \text { Department of Justice } \\ \text { DOT } & \text { Department of Transportation } \\ \text { FCC } & \text { Federal Communications Commission } \\ \text { FDA } & \text { Food and Drug Administration } \\ \text { FMC } & \text { Federal Maritime Commission } \\ \text { FPC } & \text { Federal Power Commission } \\ \text { FTC } & \text { Federal Trade Commission } \\ \text { ICA } & \text { Interstate Commerce Act } \\ \text { ICC } & \text { Interstate Commerce Commission } \\ \text { NITL } & \text { National Industrial Traffic League } \\ \text { NLRB } & \text { National Labor Relations Board } \\ \text { OMB } & \text { Office of Management and Budget } \\ \text { SEC } & \text { Securities and Exchange Commission } \\ \text { USDA } & \text { US Department of Agriculture }\end{array}$

